\section{Neue innovative}

\section{CAD/CAM-Materialien}

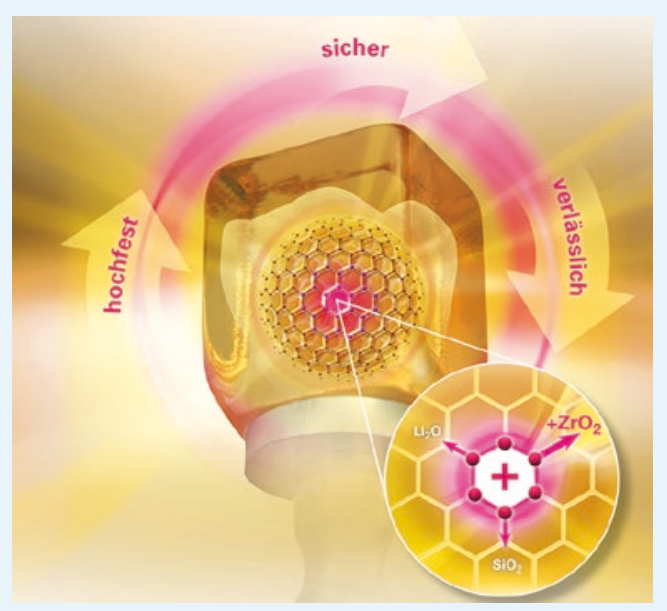

Ein wichtiger Schritt für mehr Sicherheit und Qualität in der Fertigung von Zahnrestaurationen beim Zahntechniker und Zahnarzt sind die neuen innovativen CAD/CAM-Materialien, VITA ENAMIC und VITA SUPRINITY. Auch in diesem Jahr begrüßt das VITA-Team mit zahlreichen Innovationen Zahnärzte, Zahntechniker, zahnmedizinische Fachangestellte und Zahnmedizinstudenten an insgesamt 4 Fachdental-Standorten. Vorgestellt werden die analogen und digitalen VITA-Produktlösungen für die Farbbestimmung und -kommunikation sowie Farbreproduktion und -kontrolle mit den VITA Easyshade Advance 4.0 samt der umfangreichen VITA-Serviceleistungen am 26./27. September 2014 auf der Fachdental Leipzig, am 10./11. Oktober 2014 auf der Fachdental Südwest in Stuttgart, am 18. Oktober 2014 auf der infodental süd in München und am 7./8. November 2014 auf der infodental mitte in Frankfurt a. M. Kompetent unterstützt wird das VITA-Team von ZA Knut Mau (Tuttlingen) und ZT Jens Richter (Rochlitz): Sie berichten aus Anwendersicht über ihre Erfahrungen mit VITAProduktlösungen, werden Live-Demonstrationen durchführen und vermitteln dabei viele praktische Tipps und Tricks für optimale Prozessabläufe und Ergebnisse. Durch ZT Jens Richter erfolgen außerdem 2 informative Fachvorträge über die zirkondioxidverstärkte Lithiumsilikatkeramik VITA SUPRINITY im Rahmen des Dental Tribune Study Club-Symposiums. Die Vortragszeiten sind in Leipzig 17.00 Uhr und in Stuttgart 14.00 Uhr. Weitere Informationen über die HochleistungsGlaskeramik sind erhältlich im Internet unter www.vita-zahnfabrik.com und telefonisch bei der VITA Hotline unter 07761 / 562222.

Nach einer Pressemitteilung der

VITA Zahnfabrik H. Rauter GmbH \& Co. KG, Bad Säckingen
Behandlungsstühle

\section{Erfrischend jung und} zum Verlieben schön!

Mit der neuen Design-Edition pink orchid setzt KaVo das i-Tüpfelchen auf Ihre neue ESTETICA E70 T- oder E80 T-Behandlungseinheit. Die farbenfrohen Polster in jungem, trendigen Pink und die farblich passenden Clips für die Armsysteme sind ein absoluter Hingucker und zaubern eine unverwechselbar frische, freundliche Atmosphäre in Ihr Behandlungszimmer.

Arbeiten in seiner schönsten Form, das bieten die KaVo

E70- und E80-Einheiten: Beste Ergonomie für ermüdungsfreies Arbeiten. Sichere Hygiene durch integrierte, automatisierte Hygienefunktionen und standardisierte Abläufe. Zuverlässige KaVo Qualität - der Maßstab für Sicherheit und Langlebigkeit.

Bekennen Sie Farbe, auffallend anders, und machen Sie Ihre neue KaVo-Einheit und Ihren neuen KaVo PHYSIO Evo-Behandlerstuhl zum absoluten Blickfang in Ihrer Praxis.

Nach einer Pressemitteilung der KaVo Dental GmbH, Biberach / Riss

\section{Hygiene}

\section{Für das schnelle Frischeqefühl}

Happy Morning war vor vielen Jahren die 1. Einmalzahnbürste auf dem deutschen Markt und ist seitdem laufend modifiziert und verbessert worden. Ab sofort ist die Einmalzahnbürste standardmäßig mit kurzem Bürstenkopf $(2,5 \mathrm{~cm})$ ausgestattet. Er ermöglicht einen leichteren Zugang zu den Seitenzähnen und bieten dem Anwender mehr Komfort. Die hochwertigen, abgerundeten Nylonborsten der Zahnbürste sind mit Zahnpasta imprägniert. Die selbstschäumende Eigenschaft der Zahnpasta-Imprägnierung sorgt für sofortige Einsatzbereitschaft, da kein zusätzliches Wasser benötigt wird. Dadurch ist die Bürste ideal für zwischendurch, zum Zähneputzen in der Zahnarztpraxis vor der Behandlung und auf Kurzreisen.

Die Zahnbürsten sind hygienisch einzeln verpackt, sowie mit und

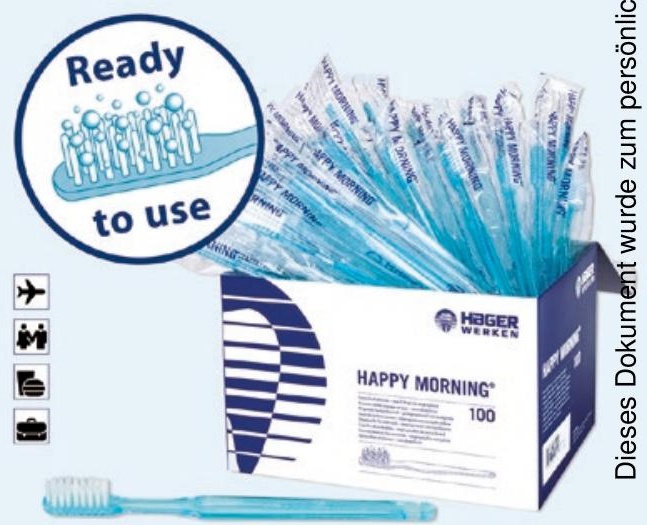

ohne Zahnpasta erhältlich. Weitere Produkte aus der Happy MorningReihe sind die Happy Morning Xylitol (Borsten mit xylitolhaltiger Zahnpasta imprägniert) und das Happy Morning Travel Kit (Zahnbürste und erfrischende Zahnpasta).

Nach einer Pressemitteilung der Hager \& Werken GmbH \& Co. KG, Duisburg www.hagerwerken.de, www.miradent.de 


\section{Schallspitzen für retrograde Wurzelkanalaufbereitung}

Sieben diamantierte Schallspitzen für die retrograde Wurzelkanalaufbereitung ergänzen die bewährte SonicLine von Komet im Rahmen der Wurzelspitzenresektion. Nach der Präparation eines Knochenfensters und der Resektion der Wurzelspitze helfen sie bei der Auffindung und Darstellung der Kanaleingänge, Präparation der Kavität, Eröffnung und Erweiterung sehr starker Wurzelkrümmungen im Frontzahnbereich und bei der Präparation des Unterschnittes zur Retention der retrograden Wurzelfüllung. Die Spitzen stehen rechts und links gebogen zur Verfügung, bieten also gute Ergonomie, um in allen Kieferbereichen optimal arbeiten zu können. Sie ermöglichen ein minimalinvasives Vorgehen ohne Präparation von großen Knochenfenstern, bieten eine achsengerechte Bearbeitung und gute Sicht. Komet empfiehlt, die neuen Spitzen im Schallhandstück SF1LM in Betrieb zu nehmen.

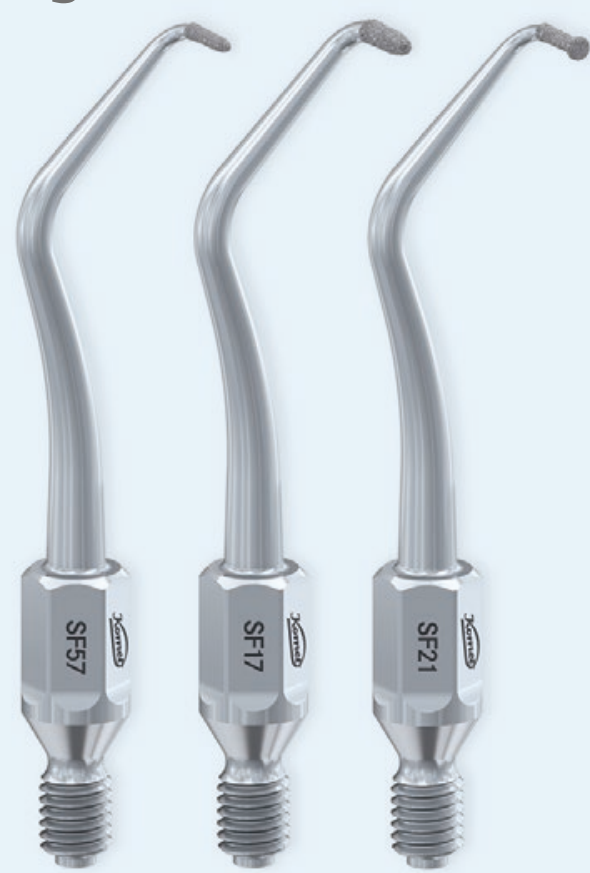

Nach einer Pressemitteilung der Komet Dental Gebr. Brasseler GmbH \& Co. KG, Lemgo

Internet: www.kometdental.de

\section{Enorme}

\section{Energieeffizienz}

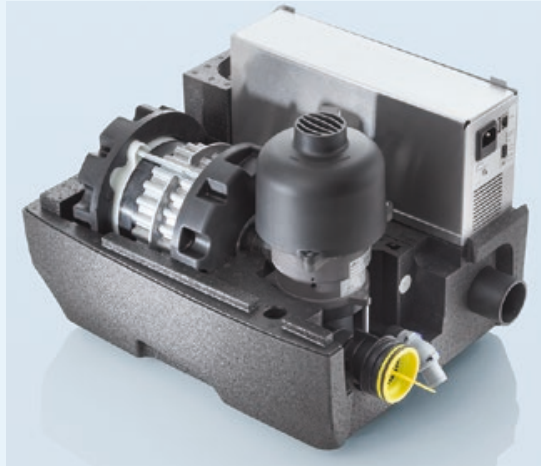

Vor 50 Jahren erreichten die Möglichkeiten zur ergonomischen Behandlung eine ganz neue Stufe - mit Saugsystemen von Dürr Dental, die eigens auf die Bedürfnisse der zahnärztlichen Praxis abgestimmt waren. Seither optimiert das Unternehmen seine Technologie kontinuierlich. Nun haben die Ingenieure darüber auf eine grundlegend neue Art und Weise nachgedacht. Die Generation der neuen dentalen Saugsysteme ist leiser, kleiner, leichter, senken den Energiebedarf um bis zu 50\% und tragen den Namen Tyscor. Statt des bekannten Seitenkanalprinzips arbeitet der Verdichter nach dem Radialprinzip. Diese Technologie erhöht die Effizienz gegenüber den bisher üblichen Saugsystemen deutlich. Bei Bedarf „verwandelt" sich ohne weiteres eine trockene Tyscor-Saugmaschine in eine nasse. Dank des modularen Aufbaus wird dabei einfach die Separierung in das bestehende System integriert. Selbstverständlich sind die neuen Saugeinheiten netzwerkfähig für die Einbindung in die Software Tyscor Pulse. Sie ermöglicht es komfortabel vom PC aus, die Saugmaschine auf 3 verschiedene Saugleistungen einzustellen (Eco, Balanced, Boost). Vor allem jedoch spart der Betreiber dank einer bedarfsgerechten Steuerung des Vakuums gegenüber herkömmlichen Geräten in der Regel bis zu 50\% Energie und damit Kosten. Ab sofort ist die Tyscor-Saugmaschinen-Generation für bis zu 2 Behandler verfügbar: in der Variante Tyscor V 2 für die trockene Absaugung und in der Variante Tyscor VS 2 für die nasse Absaugung. Für diese steht überdies der CA 2 als genau passender Amalgamabscheider zur Verfügung.

Nach einer Pressemitteilung der DÜRR DENTAL AG, Bietigheim-Bissingen Internet: www.duerrdental.com 